\title{
Los compromisos actuales de la comunicación \\ y la investigación en América Latina: Diálogo con Eva da Porta, Raúl Fuentes Navarro y Erick Torrico
}

Comunicación

número 43

Julio - diciembre 2020 | pp. 89-101

\section{Nicolás}

\section{Chalavazis Acosta}

Doctor en Filosofía de

la Universidad Pontificia

Bolivariana (UPB).

Magíster en Investigación

Psicoanalítica de la

Universidad de Antioquia.

Docente de la Facultad de Comunicación Social

- Periodismo de la

Universidad Pontificia

Bolivariana.

Correo electrónico: nicolas. chalavazis@upb.edu.co

\section{The current commitments of Communication Studies and Research in Latin America: Dialogue with Eva da Porta, Raúl Fuentes Navarro y Erick Torrico}

http://dx.doi.org/10.18566/comunica.n43.a06

Fecha de recepción: 9 de noviembre de 2020

Fecha de aceptación: 13 de noviembre de 2020

\section{Resumen}

El presente texto testimonia el lanzamiento del doctorado en Comunicación de la Facultad de Comunicación Social - Periodismo de la UPB. Aquí se compilan algunas de las principales ideas de los ponentes, que versaron acerca del campo de comunicación, del lugar que la investigación ocupa en ese campo, del pensamiento crítico y de la pertinencia de la universidad como institución.

\section{Abstract}

This articletestifies the release of the Doctorate Programme in Communication of the UPB's Faculty of Social Communication and Journalism. Here are compiled some of the main ideas of the speakers who dealt about the field of Communication and the place that Research occupies in that field, Critical Thinking and the relevance of the University as an institution.

\section{Palabras clave}

Comunicación, epistemología, pensamiento crítico, investigación, universidad.

\section{Keywords}

Communication, Epistemology, Critical Thinking, Research, University. 


\section{Introducción}

El presente texto registra algunas ideas pronunciadas durante el lanzamiento del doctorado en Comunicación de la Facultad de Comunicación Social - Periodismo de la Universidad Pontificia Bolivariana. No yace aquí una transcripción literal de lo dicho, pues no se pretendía con este corpus componer un acta que consignase los vaivenes y titubeos del sermón. Estas letras se resitúan en otro contexto, el cual determinó su estructura: se publican en la revista Comunicación, hecho que les exige un límite y las dirige a los lectores asiduos de la revista, a los buscadores de conocimiento especializado y a los navegantes incautos de la red que se topen con ellas. Así, el público se amplía y no se limita a los asistentes al evento. Algunas ideas precisaron de edición y otras de reagrupación. Así, este texto testimonia el evento con la edición que lo estructura. Como todo corte, toda edición, no es más que una intención; es decir, una sugerencia interpretativa.

\section{Contexto}

El 6 de noviembre de 2020, la UPB y su Facultad de Comunicación Social Periodismo presentaron a la ciudadanía, realizando un panel académico, el doctorado en Comunicación. Participaron la doctora Eva da Porta, ${ }^{2}$ el doctor Raúl Fuentes Navarro ${ }^{3}$ y el doctor Erick Rolando Torrico Villanueva, ${ }^{4}$ y moderó el coordinador del doctorado, el doctor Juan Carlos Ceballos Sepúlveda. Este evento sirvió, además, de preámbulo a la apertura del XV Congreso Internacional ALAIC ${ }^{5}$ 2020, cuyo país anfitrión y organizador fueron Colombia y la mencionada facultad.

El evento fue virtual y aconteció durante tiempos del confinamiento obligado por la pandemia de COVID-19, lo cual le confirió, además de su valor académico intrínseco, una relevancia ética que pretextó la reflexión acerca del valor de la tecnología en el campo de la comunicación, la persistencia ética de la universidad y su vigencia como institución en Occidente.

\section{Intervenciones y reflexiones institucionales}

El rector general, presbítero y magíster Julio Jairo Ceballos, reconoció los esfuerzos de los docentes que concretaron el doctorado y justipreció el valor epistemológico de la comunicación.

El decano de la Escuela de Ciencias Sociales, magíster Ramón Arturo Maya Gualdrón, destacó, en calidad de historiador, que el doctorado fue posible gracias a la madurez intelectual e incumbencia histórica de una Facultad de

Comunicación

número 43

julio - diciembre

2020
1 Para quien desee ver todo el evento, puede consultarlo en el canal de YouTube de la universidad: Universidad Pontificia Bolivariana (2020), Presentación del doctorado en Comunicación (https: / / tinyurl. com/y5jjw9jh).

2 Doctora en Comunicación de la Universidad Nacional de La Plata, Argentina, y docente e investigadora del Centro de Estudios Avanzados de la Facultad de Ciencias Sociales de la Universidad de Córdoba, Argentina.

3 Doctor en Ciencias Sociales, Profesor emérito del ITESO, Instituto Tecnológico y de Estudios Superiores de Occidente y Profesor titular del Departamento de Estudios de la Comunicación Social de la Universidad de Guadalajara.

4 Doctor en Comunicación por la Universidad Rey Juan Carlos en Madrid, director académico del postgrado en Comunicación y Periodismo de la Universidad Andina Simón Bolivar, en La Paz (Bolivia), y docente investigador de ciencias de la comunicación en la Universidad Mayor de San Andrés.

5 Asociación Latinoamericana de Investigadores de la Comunicación. 
Comunicación Social - Periodismo que ha estado formando profesionales integrales con alta calidad académica durante 54 años y que ha ido componiendo y legando un saber entre sus docentes ininterrumpidamente. Esta madurez, justamente, permitió la transformación curricular vigente. Mencionó la importancia de los docentes fundadores que ya no están en la facultad, quienes siguen presentes como tradición.

Un doctorado es una institución "superior para lo superior", dijo, cuyo impacto repercute en la sociedad y se inicia en los procesos investigativos soportados en los grupos GICU, ${ }^{6}$ Gescom ${ }^{7}$ y Epilión, ${ }^{8}$ y que deben propender hacia la construcción de redes de investigación de carácter internacional. Por último, recordó la importancia de la ética para continuar formando seres humanos autónomos y críticos, de manera que en la sociedad haya "equidad, justicia y la alegría de la vida".

La directora de la facultad, magíster María Victoria Pabón Montealegre, preponderó los verbos "profundizar, investigar, conocer, preguntar y transformar" sobre el verbo "reinventarse" para figurar una idea del espíritu y de la identidad del doctorado y de la facultad: aquellos no desconocen el pasado, sino que atienden los cambios del presente y se fijan siempre en lo humano y lo social. En consonancia con lo expresado por el decano, recalcó la madurez y el recorrido de la facultad. Recordó, además, que el doctorado es producto de una estrategia que promovió la formación doctoral de 15 de sus docentes, lo cual afianzó las redes investigativas y teóricas que soportarán las líneas de formación del doctorado en Comunicación: ciudadanías, organizaciones, apropiaciones y narrativas.

Las ciudadanías, clarificó, se dicen en plural porque su riqueza estriba en la heterogeneidad cultural y política. Las organizaciones son redes humanas atravesadas por el lenguaje e interconectadas con personas, grupos internos o externos a ellas. Las apropiaciones suponen una incumbencia que permite usos, prácticas sociales, modos de valorar y hábitos que derivan en que el ser humano sienta algo como propio. Las narrativas obedecen a la multiplicidad de lenguajes, son campos mutables que posibilitan la expresión de las ideas que permiten hacer mundos, cambiar los mundos y transformar la sociedad.

Insistió en la importancia del contexto, situando la función social del doctorado en el marco en el que surge: una Colombia desigual y conflictiva, aunque habitada por un pueblo resiliente, trabajador y alegre que merece un mejor futuro. Declaró con un tono académico y ánimo integrador:

No es cuestión del azar que en menos de cuatro años hayan surgido cinco programas doctorales de comunicación en Colombia. Esto habla de la gestión académica e investigativa que se desarrolla en los claustros de nuestro país,
6 Grupo de Investigación en Comunicación Urbana.

7 Grupo de Investigación en Gestión de la Comunicación. 8 Grupo de investigación en publicidad. 
y que, seguramente en unos años, confiamos, contribuya a la integración de Latinoamérica para el estudio de la comunicación.

Añadió que el doctorado anhela formar investigadores interesados en las inestabilidades sociales del país y de América Latina. Expuso sobre la necesidad de reflexionar la identidad del latinoamericano y el lugar de las tecnologías en la sociedad, pues cada vez más marcan nuevas maneras de relación, trabajo y participación social. Solicitó no olvidar las reflexiones teóricas de la comunicación latinoamericana que brotaron con fuerza en las décadas de los 70 y 80, tristemente abandonadas en años recientes, pues en ellas yacen proyecciones y pistas para pensar la realidad actual sin desconocer la novedad del presente. Debe promoverse, enfatizó, la formación de investigadores que sepan leer el mundo globalizado y aportar a las particularidades de la región. Instó a trabajar entre pares y a revisar constantemente el conocimiento.

Evocó los años de discusiones académicas que asumió la facultad para lograr no solo el doctorado (el cual se "cocinó a fuego lento"), sino también su transformación curricular. Aquí la lentitud, que no tardanza, constituye un motivo de orgullo, pues demuestra una reflexión responsable. La facultad se comprehende como un sistema completo e integral donde todos aportan y se enriquecen.

Tener claridad sobre nuestro punto de inicio, el pregrado, y nuestro punto de llegada, el doctorado, nos permite apostarle a un comunicador que en su nivel de profesionalización sea sensible, lea y conceptualice el mundo, lo narre; en su nivel de especialización y maestría, le aporte cambios a ese mundo para que, en el doctorado, investigue, genere nuevo conocimiento y metodologías, de tal forma que se complete el sistema.

Por último, agradeció a los profesores que lideraron el proyecto doctoral: las doctoras Beatriz Marín, Verónica Suescún, Ana María López y Érika Jaillier, así como los doctores Ómar Muñoz y Juan Carlos Ceballos.

También intervinieron algunos docentes de la facultad:

La especialista Luz Gabriela Gómez Restrepo, quien fue decana (así se conocía otrora la dirección de la facultad), expresó que el "doctorado llega en un momento muy bello", y espera que sirva para ser más sabios y mejores seres humanos. "Los proyectos académicos tienen que permitirnos crecer en humanidad", pues "enseñar y estudiar son verdaderos oficios de paz".

La doctora Beatriz Marín destacó las potencias académicas del doctorado argumentando que quien lo estudiare se encontrará con lo siguiente: a) 
el sello de la universidad, su humanismo cristiano; b) el respaldo de la Escuela de Ciencias Sociales, la cual durante 75 años se ha destacado en su investigación y proyección social; c) los soportes tanto de la Facultad de Comunicación Social - Periodismo como de una Facultad de Publicidad que aporta categorías de investigación y evaluación, así como muestra otras metodologías y objetos de estudio, como son la creatividad, el consumo y el entorno; d) los grupos de investigación del programa, las líneas de formación, las redes de investigación, como ALAIC y Acicom, ${ }^{9}$ y las redes académicas, como Felafacs, ${ }^{10}$ Afacom, ${ }^{11}$ Claep, ${ }^{12}$ Confibercom, ${ }^{13}$ entre otras.

El magíster Federico Medina Cano, quien hasta hogaño ha sido profesor de Semiología, Teorías de la Comunicación y Literatura, expuso que el doctorado revela un proceso de madurez y decantación de sus líneas de reflexión académica; además, insistió en que permite a los egresados del pregrado, especialización y maestrías profundizar en la noción de realidad social y de entorno, así como la concienciación de las demandas del medio, de los actores que intervienen en él.

La doctora Carmen Victoria Restrepo, docente de Investigación y Teorías de la Comunicación, resaltó el reto investigativo que implica el doctorado y los rigores que supone llevar a los docentes, estudiantes y objeto de estudio, la comunicación, al más alto nivel.

El profesor emérito Juan José García Posada expuso que el doctorado enriquece la cultura profesional del periodismo y de la comunicación, cuya estructura se basa en tres columnas: a) la fundamentación filosófica y ética, b) la innovación metodológica y c) el manejo de la tecnología. Señaló que mientras la reflexión teórica y ética está rezagada, ha habido un desarrollo tecnológico acelerado, por lo cual instó a que un doctorado en Comunicación empareje las cosas. Así mismo, declaró que un doctorado tiene el reto de demostrar la calidad de un programa que ha sido acreditado y reacreditado nacional e internacionalmente.

\section{Diálogo con Eva da Porta, Raúl Fuentes Navarro y Erick Torrico}

El coordinador del doctorado, doctor Juan Carlos Ceballos, presentó a los asistentes y recalcó su relevancia teórica en el ámbito de la comunicación. Enfatizó el título del panel, "Los compromisos actuales de la comunicación y la investigación en América Latina en el marco del lanzamiento de un doctorado", para situarlo dentro de un contexto colombiano y latinoamericano que reta a los doctores y estudiosos de un doctorado, y, por último, reconoció que este proyecto traerá problemas teóricos y retos que han de asumirse con múltiples abordajes.
93

comunicación

número 43

julio - diciembre

2020
9 Asociación Colombiana de Investigadores en Comunicación.

10 Federación Latinoamericana de Facultades de Comunicación Social.

11 Asociación Colombiana de Facultades y Programas Universitarios de Comunicación. 12 Consejo Latinoamericano de Acreditación de la Educación en Periodismo.

13 Confederación Iberoamericana de Asociaciones Científicas y Académicas de Comunicación. 
A cada panelista se le formuló una pregunta. Si bien cada teórico expuso desde su perspectiva, hubo algunos temas coincidentes dignos de mención: a) los lugares epistemológico y ético de la comunicación, b) el pensamiento crítico, c) la universidad como institución en la actualidad, d) la situación de América Latina en el campo de la comunicación y e) las responsabilidades y relevancia de un doctorado en Comunicación.

\section{Doctora Eva da Porta}

El doctor Ceballos le preguntó: con respecto a la investigación, ¿en qué condiciones se da la investigación en nuestros contextos latinoamericanos y cuál es el rol de los investigadores en ellos?

Luego de agradecer, la doctora Da Porta felicitó públicamente al doctor Raúl Fuentes por el reconocimiento de doctor honoris causa que le confirió la Universidad de Baja California. Declaró que su exposición se ajustaría a su contexto y preocupaciones epistemológicas e intentó responder a la pregunta formulada. Su respuesta, afortunadamente, abarcó mucho más de lo preguntado. He aquí algunas de sus ideas:

\section{El proyecto doctoral}

La doctora se refirió al proyecto doctoral entendiéndolo como el conjunto de discusiones que lo concretaron y le brindaron una situación epistemológica. En primer lugar, reconoció el reto práctico que supone generar tal proyecto; esto es, la elección de un proyecto y de un campo.

Entre un proyecto y su desarrollo hay una distancia grande y, muchas veces, debido a las contingencias de la vida, el doctorado puede desviarse paulatinamente del ánimo que lo concretó. La fundación ha de ser un lugar del que no hay que alejarse y al que siempre hay que retornar. Entonces, instó al doctorado a no olvidar su misión ni las discusiones que lo produjeron.

Las prácticas y el contacto con la realidad son importantes, pues marcan nuevos rumbos, y así el proyecto doctoral no se queda en letra muerta. Hay que ir del proyecto a la práctica y de la práctica al proyecto.

\section{La investigación como modus vivendi académico}

Los doctorados en Comunicación y Ciencias Sociales tienen dos propósitos centrales: formar e investigar. Estas misiones no pueden pensarse de manera aislada. No solo debe formarse a los doctorandos, sino incitarlos a pensarse como investigadores, aun en el contexto arduo de América Latina. 
Denunció el problema, y lo reconoció como reto, de que la mayoría de las universidades latinoamericanas no permiten fácilmente centros exclusivos, sistemáticos, críticos y reflexivos dedicados solo a la investigación, y especialmente a la comunicación.

La investigación, más que un formalismo, debe estar implantada desde el comienzo del doctorado. "Ojalá los estudiantes vinieran con formación de investigadores desde antes". A investigar se aprende investigando al lado de otros, primero con labores simples y, luego, complejas. No debe llegarse al final de la carrera sin formación investigativa. El investigador debe nutrirse de las investigaciones de los demás y debe enriquecer al propio doctorado. Es importante incitar a los estudiantes a que se presenten a becas para que tengan tiempo de investigar, pues ello requiere de mucha dedicación y los problemas económicos suelen ser causa de alejamiento de las investigaciones.

\section{Estímulo de las publicaciones y las relaciones públicas}

Deben estimularse las publicaciones y ponerse a la luz pública porque ser un académico exige ser criticado, ser revisado y poder debatir. La ciencia es una producción intersubjetiva que necesita de la mirada ajena. La participación en congresos debe ser una cuestión activa y primordial del doctorado.

\section{Las tecnologías}

Las redes y las tecnologías son una posibilidad. La época de pandemia lo demuestra más que nunca. Con aquellas se puede seguir produciendo y trabajando. El doctorado debe permitir las tecnologías y trabajar colaborativamente con ellas.

\section{Cuestión epistemológica del campo de la comunicación en América Latina}

¿Desde qué lugar pensar un doctorado que se enfoca desde América Latina y en América Latina? No hay una sola respuesta. Esta debe ser la pregunta epistemológica de este doctorado. En ese sentido, cuando se dice que una carrera se plantea en América Latina y desde América Latina, se declara una posición geográfica, pero también una apuesta investigativa. El doctorado debe reflexionar sobre el modo en el que se investiga en la región y en sus potencialidades.

No debe tenerse una visión cerrada del campo, pues en la academia el diálogo es alimento: "Nos alimentamos del diálogo con las otras disciplinas". 
Ha de trabajarse inter- y transdisciplinariamente: esto fortalece y enriquece el doctorado y el campo de estudio.

América Latina debe ser entendida como una pregunta investigativa y no como un paquete cerrado de respuestas. Es necesario trabajar con una pregunta que permita recuperar la memoria del campo, recuperar el presente y proyectar sus líneas investigativas hacia el futuro.

\section{La tradición como lugar}

El presente permite revisitar el pasado:

Tenemos un pasado muy rico. Tenemos un buen momento en el presente para ver cómo esas voces fundacionales del pasado nos hacen preguntas actuales; por eso son pensamientos fundacionales. Celebro la posibilidad de que se recuperen las voces de los padres fundadores como Paulo Freire, Ramiro Beltrán, de Pascualli, y hay que mostrar qué hicieron, qué preguntas nos dejaron. Es central, al recuperar la memoria, que cuestionemos el presente y recuperemos autores que nos siguen resonando y que nos permiten pensar la investigación: Martín Barbero, García Canclini, López, Eliseo Verón, Rosana Reguillo, Fuentes Navarro, Orozco Gómez, Vasallo de López, Torrico... Mas no como letra sagrada, escrita y acabada.

A los pensadores se los honra si se los recupera de modo crítico. La tarea de un doctorado en Comunicación es recrear aquello heredado y proponer nuevos destinos en un diálogo que tiene que ser provocado.

\section{Pensamiento crítico}

América Latina ha pensado y sigue pensando críticamente. El pensamiento crítico aparece cuando hay una crisis en la episteme. La región y la teoría han venido de crisis en crisis epistémica. Eso abre la posibilidad de un pensamiento crítico, que no supone un consenso consigo mismo. Debe repensarse críticamente la realidad que se investiga y, sobre todo, cómo se piensa en un lugar propio, es decir, refiriéndose al contexto. El pensamiento crítico no puede ser un protocolo sagrado, sino una pregunta transformadora.

El pensar crítico lleva a lugares incómodos de cuestionamiento, pone en crisis los sentidos comunes y desnaturaliza lo usual, supone ir a contracorriente. Como ese lugar es incómodo, necesita argumentarse, justificarse, ser reflexivo y dar cuenta de las decisiones. El pensamiento crítico es un desafío a lo existente, en el sentido de que esto último, la "realidad dada", pareciera habérsele dado a la sociedad como inmutable y eternal. Han de ponerse en tensión, en el pensar crítico, el saber y el poder. 
El pensamiento crítico es una práctica que debe llevarse a cabo en la investigación desde sus inicios cuando se piensa el problema investigativo y ha de mantenerse hasta en los modos en los que el investigador se vincula con los demás y se acerca a la realidad; implica una práctica que pone en juego la dimensión ética, siendo un pensamiento que no es normalizado y que debe reflexionar sobre sí mismo constantemente. La mejor forma de permitir la crítica es el sostenimiento del diálogo y la apertura del campo a otras disciplinas sin temer la pérdida de rigor de la disciplina misma.

¿Cómo se tejen los hilos del poder? No puede tenerse una mirada ingenua ante el poder, hay una labor política en cuanto a cómo se lo lee y se lo asume. Un pensamiento crítico debe atender el lenguaje, pues los actos de poder se dan allí. Estamos hechos del lenguaje. El lenguaje no es ingenuo: cada palabra y categoría se ubica en redes de sentido y sugiere redes de interpretación. Hay que asumir atenta y amorosamente el lenguaje que se lee y en el que se transmite.

Para culminar, se apoyó en la cita de Héctor Schmucler:

Me parece que cuando se habla desde el pensamiento crítico se apunta a esto: estar permanentemente atentos, a ver que nuestro pensar está constituido por la suma de múltiples personas, que no estamos empezando casi nada nuevo. Me refiero a la idea de diálogo y esto, a veces, resulta provocador.

\section{Doctor Raúl Fuentes}

El doctor Ceballos retomó el tema del pensamiento crítico. La universidad - dijo- como institución social debe crear unas condiciones para propiciar el pensamiento incómodo que nos lleve a pensar en la producción de contenidos y conocimiento.

Le preguntó al doctor Fuentes: ¿cuál es el papel de la universidad en este contexto y qué condiciones se necesitan desde allí para propiciar discusiones y generar conocimiento?

He aquí algunas de sus ideas:

\section{Persistencia y compromiso de la universidad}

Las universidades han cerrado sus puertas por la pandemia, pero no han dejado de trabajar. Ellas han de seguir laborando, pues tienen un compromiso político y educativo. Hemos aprendido a cuestionar nuestra propia certeza y sobre las cuestiones en las que trabajamos. ¿En qué 
podemos basar nuestra capacidad de decir algo e intervenir sobre la interpretación social de cualquier acontecimiento? Ahora, con todo lo que ha sucedido en el mundo, especialmente este año (lo que ha pasado este año no es totalmente inédito o nuevo, la realidad social en América Latina se mantiene: la desigualdad social, la insuficiencia de recursos educativos, etcétera), la universidad ha tenido y sigue teniendo un lugar estratégico, fundamental y constitutivo en las ciudades de todo el mundo.

Conviene reconocer de entrada que América Latina es heterogénea. Nuestra historia tiene una perspectiva a mediano plazo en términos del avance de las estructuras mundiales. La misión de las universidades en la región es, sobre todo, una posición de cuestionamiento y distanciamiento para aportar una mirada reflexiva y crítica que pueda ayudar a la sociedad, que formule sus retos y cuestiones fundamentales, para advertir las razones por las cuales el entorno social se afecta y cómo podría enfrentarse la búsqueda de soluciones a esas problemáticas en todos los campos. Esto a veces es difícil de distinguir porque las universidades no se aíslan de la sociedad; al contrario, se confunde su tarea con la participación de los repartos ideológicos, económicos y de los recursos de la sociedad para fines particulares, sectoriales, para fines egoístas. Esa tensión exige que dentro de la universidad se mantenga el espíritu de cuestionamiento de la mejor manera, más productiva, más útil... ¿Cuál es el sentido de lo que se hace y el sentido de lo que se está interpretando? Es misión de un programa de investigación y de formación, como es el caso de un doctorado, responder a esa cuestión.

Hay análisis que muestran que en América Latina hay muchas instituciones de educación superior que tienen autoridad formal para expedir títulos, pero eso no las legitima como universidades. La personalidad jurídica no hace a una universidad. En realidad, en América Latina hay pocas universidades que desarrollen la investigación y formen críticamente, y, además, son pocas las que colaboran entre ellas y los sectores sociales a los que se deben. Se espera que las universidades dialoguen en relación directa y cercana. Eso se traduce como compromiso con la verdad y la realidad.

\section{El equilibrio entre el rigor y la imaginación}

Subrayó el carácter de un investigador no latinoamericano: el sociólogo estadounidense Andrew Abbott, quien concibe el trabajo científico como una tensión permanente entre el rigor y la imaginación; esa tensión, que antes solía llamarse contexto del descubrimiento y contexto de la justificación, es pertinente para recuperar la reflexión de la comunicación en América Latina.

¿Cómo se ha asumido en la región esta tensión? Estamos en un momento histórico en el cual ella debe verse reforzada porque parece que la imaginación 
corre un riesgo en detrimento del rigor; en efecto, sin el contrapeso de la imaginación, el rigor no encontraría tensión y podría convertirse en solo peso neto, en tiranía, en verdad indiscutible, en imposibilidad de imaginar.

El reto fundamental de este programa, y de todos los programas, es asumir la imaginación comprometida con la práctica social, no entendida como la de la fantasía literaria, sino como aquella que permita descubrir los factores de una situación, de un contexto, para que la sociedad tenga un futuro mejor, uno más adecuado. Este doctorado puede ofrecer eso mejor que otras disciplinas. Debe ser, entonces, una tarea de gremio. No puede ser tarea de individuos: precisa de debates, de diálogos y de colaboraciones.

\section{Doctor Erick Torrico}

Preguntó el coordinador, doctor Ceballos: ¿cuáles son los retos y las problematizaciones que nos brinda la perspectiva latinoamericana de la comunicación?

He aquí algunos aspectos de la disertación del doctor Torrico:

\section{Función de un doctorado}

Un programa de doctorado busca motivar la investigación, cultivar el dominio crítico y las interpelaciones del saber; tiende a alentar tanto la creación como la discusión del conocimiento. Podría decirse que su propósito es formar intelectuales críticos, especialistas en unos temas concretos, pensadores dotados de solvencia académica que puedan incidir en el ámbito público en función de unos criterios. Un doctorado surge en un contexto donde haya necesidad social e institucional de un proceso formativo de esa naturaleza para ofrecerles conocimiento y alternativas de respuesta a la ciencia y a la práctica. El doctorado responde a la necesidad de dar respuesta intelectual a los vacíos del conocimiento o a urgencias sociales.

\section{Estudio de la comunicación en América Latina}

Hace falta considerar dentro de nuestro contexto una sociedad regional. Hay mucho por decir, tomando en cuenta la heterogeneidad de la subregión.

El repaso de algunos trazos históricos del campo de la comunicación puede ser útil, puesto que de él se derivan algunos problemas y desafíos sobre los que vale la pena reflexionar: 
Aunque estudiosos como el boliviano Luis Ramiro Beltrán y el brasileño José Marques de Melo documentaron trabajos precursores de la comunicación en la realidad latinoamericana desde la segunda mitad del siglo XIX, u otros trabajos hablan de la prehistoria de la investigación del campo de la comunicación entre los años 30-50 del pasado siglo, no es sino hasta las décadas de los 60 y 70, como lo ha establecido Raúl Fuentes en varios de sus trabajos, cuando esta labor de investigación especializada despega para irse consolidando con la constitución de la Asociación Latinoamericana de Investigadores de la Comunicación. Luego, en la década del 90, llegan a proliferar las facultades de comunicación y justo allí se le presta mucha más atención a dicho campo.

En el presente, el establecimiento de programas de postgrado resulta fundamental. La investigación en América Latina ha ido evolucionando y ha ido elaborando un perfil propio que se distingue por su sentido crítico, el interés popular, la identidad regional, el diálogo y el desarrollo de la democracia. Este despliegue y estas categorías investigativas han llevado a creer que Latinoamérica es un bloque homogéneo, mas no es verdad. No todo lo que se hace en la investigación en Latinoamérica persigue un mismo norte.

Pueden clasificarse los estudios latinoamericanos en comunicación según su carácter ante el saber heredado:

1. Línea de reproducción. Solo imita o repite los métodos de moda en la investigación comunicacional que se dan en otras latitudes.

2. Línea de análisis. ${ }^{14}$ Examina, categoriza y ratifica los conceptos, los temas y, a veces, hasta las conclusiones de las corrientes investigativas importadas.

3. Línea de renovación. De cuando en cuando, aporta elementos diferenciadores sin llegar a una ruptura abierta con los modelos predominantes.

4. Línea de innovación. De forma extraordinaria puede llegar a plantear visiones novedosas y distintas de las ya conocidas.

Esta clasificación es compatible con los tres grados de observación científica que el filósofo colombiano Castro Gómez identifica en su ejercicio de cuestionamiento de la racionalidad latinoamericana, que él encuentra sujeta a los parámetros de la modernidad occidental:

1. La mera aplicación de las formas positivistas cuya presunta universalidad se asume sin reflexión.

2. El contraste de lo observado en los fenómenos con estas formas positivistas, que puede llegar a ser contrario, pero que se mantiene dentro de los límites que estos parámetros establecen.
14 El audio de la charla del doctor Torrico presentó muchos problemas de sonido, cortes y saltos de imagen; así que dedujimos el nombre de esta línea de orientación de vocablos de la explicación misma; desde luego, bien pudiera ser otro. 
3. El orden del saber desde el que son hechas las observaciones extrarregionales.

A estos tres grados habría que añadirles un cuarto: la construcción de un lugar propio que postula una epistemología, una historia y una teoría distintas de las que predominan con pretensiones de universalidad.

De todo lo dicho se desprende un problema, lo que puede ser llamado como la condición subsidiaria de la comunicación latinoamericana a la comunicación occidental, que surge de las realidades, de los intereses y perspectivas del eje que proclama su validez global.

Se deriva de todo un reto cardinal: investigar la comunicación desde la memoria y las circunstancias históricas de la región. Estos elementos deben considerarse en el programa del doctorado que ahora se inicia. Los intelectuales críticos deben sentir y actuar desde ahí. 